\title{
SVD on Intersection Spaces
}

\author{
Mazen Ali ${ }^{\dagger}$ and Anthony Nouy ${ }^{\ddagger}$ \\ † Ulm University, mazen.ali@uni-ulm.de \\ $\ddagger$ Centrale Nantes, LMJL, anthony.nouy@ec-nantes.fr
}

\section{Introduction}

I. For a linear, bounded and compact operator $T \in \mathcal{K}\left(H_{2}, H_{1}\right)$ between Hilbert spaces $H_{2}$ and $H_{1}$ the spectral theorem tells us

(SVD) $\quad T w=\sum_{k=1}^{\infty} \sigma_{k}\left\langle w, h_{k}^{2}\right\rangle_{H_{2}} h_{k}^{1}, \quad \forall w \in H_{2}$

for orthonormal $\left\{h_{k}^{1}\right\}_{k},\left\{h_{k}^{2}\right\}_{k}$ and positive nonincreasing $\left\{\sigma_{k}\right\}$ with $\lim _{k} \sigma_{k}=0$.

The space $H=\left(H_{1} \otimes H_{2},\langle\cdot, \cdot\rangle_{H}\right)$ equipped with the canonical inner product

$\left\langle v_{1} \otimes w_{1}, v_{2} \otimes w_{2}\right\rangle_{H}=\left\langle v_{1}, v_{2}\right\rangle_{H_{1}} \cdot\left\langle w_{1}, w_{2}\right\rangle_{H_{2}}$

is a Hilbert space. An algebraic tensor $u=$ $\sum_{k=1}^{r} v_{k} \otimes w_{k} \in H_{1} \otimes_{a} H_{2}$ can be identified with an operator via

$$
T_{u}(w)=\sum_{k=1}^{r}\left\langle w, w_{k}\right\rangle_{H_{2}} v_{k}
$$

Property (2) implies $\left(\left\{h_{k}^{1}\right\}_{k}\right.$ and $\left\{h_{k}^{2}\right\}_{k}$ orthonormal $\Rightarrow\left\{h_{k}^{1} \otimes h_{m}^{2}\right\}_{k, m}$ orthonormal . Then, $\|u\|_{H}=\left\|T_{u}\right\|_{\mathrm{HS}}$ and $H=\operatorname{HS}\left(H_{2}, H 1\right)$.

SVD from (1) applied to $u$ provides an optimal low rank approximation. Example: $H=L_{2}\left(\Omega_{1} \times \Omega_{2}\right)$.

II. For $H=H^{1}\left(\Omega_{1} \times \Omega_{2}\right)$

$$
H^{1}\left(\Omega_{1} \times \Omega_{2}\right) \not \subset \mathcal{K}\left(H^{1}\left(\Omega_{2}\right), H^{1}\left(\Omega_{1}\right)\right) .
$$

Here SVD does not apply.

The Sobolev $H^{1}$ is isomorphic to the intersection space

$$
\begin{aligned}
H^{1}\left(\Omega_{1} \times \Omega_{2}\right) & =\left(H^{1} \otimes L_{2}\right) \cap\left(L_{2} \otimes H^{1}\right) \\
& =: H^{10} \cap H^{01} .
\end{aligned}
$$

SVD applies on both $H^{10}$ and $H^{01}$. For $u \in H^{1}$

$$
\begin{aligned}
& u=\sum_{k=1}^{\infty} \sigma_{k}^{10} x_{k}^{1} \otimes y_{k}^{0} \quad \text { in }\|\cdot\|_{10}, \\
& u=\sum_{k=1}^{\infty} \sigma_{k}^{01} x_{k}^{0} \otimes y_{k}^{1} \quad \text { in }\|\cdot\|_{01} .
\end{aligned}
$$

For details see [1].

\section{References}

[1] W. Hackbusch. Tensor spaces and numerical tensor calculus. Springer Series in Computational Mathematics, 42:xxiv +500, 2012 .

[2] L. Grasedyck. Hierarchical singular value decomposition of tensors. SIAM J. Matrix Anal. Appl. 31:2029-2054, 2009/10.

[3] M. Bachmayr, W. Dahmen. Adaptive low-rank methods: Problems on Sobolev spaces. SIAM J. $\mathrm{Nu}$ mer. Anal., 54:744-796, 2016.

\section{Acknowledgements}

The collaboration between Mazen Ali and Anthony Nouy was supported by the European Model Reduction Network (EU-MORNET).

\section{Theoretical Perspective}

I. Consider the finite dimensional subspaces

$$
U_{r}^{10}:=\operatorname{span}\left(x_{k}^{1}: 1 \leq k \leq r\right), \quad U_{r}^{01}:=\operatorname{span}\left(y_{k}^{1}: 1 \leq k \leq r\right),
$$

and the $H^{1}$ orthogonal projections $P_{r}: H^{1}\left(\Omega_{1}\right) \rightarrow U_{r}^{10}$ and $Q_{r}: H^{1}\left(\Omega_{2}\right) \rightarrow U_{r}^{01}$. One can show

Theorem 1. Assume additionally $u \in H^{2}\left(\Omega_{1} \times \Omega_{2}\right)$. This implies $U_{r}^{10} \subset H^{2}\left(\Omega_{1}\right)$ and $U_{r}^{01} \subset$ $H^{2}\left(\Omega_{2}\right)$. Define the regularity measures

$$
L(r)=\sup _{v \in U_{r}^{10}} \frac{\|v\|_{1}}{\|v\|_{0}} \cdot \sup _{v \in U_{r}^{10}} \frac{\|v\|_{2}}{\|v\|_{1}}, \quad R(r)=\sup _{v \in U_{r}^{01}} \frac{\|v\|_{1}}{\|v\|_{0}} \cdot \sup _{v \in U_{r}^{01}} \frac{\|v\|_{2}}{\|v\|_{1}} .
$$

Then, we get

$$
\left\|u-\left(P_{r} \otimes Q_{r}\right) u\right\|_{1} \leq\left(\sum_{k=r+1}^{\infty}\left(1+r L^{2}(r)\right)\left(\sigma_{k}^{10}\right)^{2}+\left(1+r R^{2}(r)\right)\left(\sigma_{k}^{01}\right)^{2}\right)^{1 / 2} .
$$

More generally, for $d>2$, applying HOSVD ([2]) w.r.t. (a generalized) $\|\cdot\|_{\text {mix } \text {-norm }}$

$$
\left\|u-\left(P_{r}^{1} \otimes \cdots \otimes P_{r}^{d}\right) u\right\|_{1} \leq(\sqrt{r} C(r))^{d-2}\left(\sum_{j=1}^{d} \sum_{k=r+1}^{\infty}\left(\sigma_{k}^{j}\right)^{2}\right)^{1 / 2} .
$$

II. Applying SVD to coefficients. Consider a $2 \pi$-periodic function $u \in H^{1}\left([-\pi, \pi]^{2}\right)$. Then

$$
\begin{aligned}
u & =\frac{1}{2 \pi} \sum_{k, m \in \mathbb{Z}} c_{k m} e^{i k x} e^{i m y}=\frac{1}{2 \pi} \sum_{k, m \in \mathbb{Z}} c_{k m} \sqrt{1+k^{2}+m^{2}} \frac{1}{\sqrt{1+k^{2}+m^{2}}} e^{i k x} e^{i m y} \\
& \approx \frac{1}{2 \pi} \sum_{l=1}^{\infty} \sigma_{l} \sum_{\nu \in \mathbb{Z}}\left(\sum_{k \in \mathbb{Z}} E(k, \nu) v_{k}^{l} e^{i k x}\right)\left(\sum_{k \in \mathbb{Z}} E(m, \nu) w_{m}^{l} e^{i m y}\right)
\end{aligned}
$$

where $E$ are exponentials. Similar was applied in [3].

III. Consider the direct sum space $H_{2 D}:=H^{10} \times H^{01}$. We get a natural interpretation for $u \in H^{1}$.

Theorem 2. The Sobolev space $H^{1} \hookrightarrow H_{2 D}$ can be isometrically embedded into $H_{2 D}$ via $u \mapsto$ $(u, u)$. This gives the best low rank approximation as

$$
\inf _{\substack{v \in H^{10}, \operatorname{rank}(v) \leq r, w \in H^{01}, \operatorname{rank}(w) \leq r}}\|u-(v, w)\|_{2 D}=\left\|u-\left(\left(P_{r} \otimes i d\right) u,\left(i d \otimes Q_{r}\right) u\right)\right\|_{2 D} .
$$

Moreover, interpreting $u$ as an operator in $H S\left(L_{2}\left(\Omega_{1}\right) \times H_{1}\left(\Omega_{2}\right), H^{1}\left(\Omega_{1}\right) \times L_{2}\left(\Omega_{2}\right)\right)$ we obtain the SVD

$$
u=\sum_{k=1}^{\infty} \sigma_{k}^{\cup} x_{k} \otimes y_{k}, \quad x_{k} \otimes y_{k}= \begin{cases}\left(x_{l}^{1}, 0\right) \otimes\left(y_{l}^{0}, 0\right), & \text { if } \sigma_{k}^{\cup}=\sigma_{l}^{10}, \\ \left(0, x_{l}^{0}\right) \otimes\left(0, y_{l}^{1}\right), & \text { if } \sigma_{k}^{\cup}=\sigma_{l}^{01} .\end{cases}
$$

\section{Numerical Experiments}


Figure 1: Low rank approximations for truncated Fourier series of $u(x, y)=\left(x^{2}+y^{2}\right)^{0.3}(1,1)$ and $u(x, y)=$ $|x+y|^{0.6}(1,2)$. Galerkin solutions for reference functions $u(x, y)=\exp (\cos (x) \cos (y))(2,1)$ and $u(x, y)=$ $\left|1-\left(x^{2}+y^{2}\right)\right|^{0.95}(2,2)$. 